LÍVIA ADRIANO VIERA

Sônia Valadares Lemos Silva ${ }^{2}$

Roberto BUENFIL DE FARIA ${ }^{3}$

UMBERTO GAZI LIPPI ${ }^{3}$

Reginaldo Guedes Coelho Lopes ${ }^{1}$

Artigo Original

Palavras-chave

Medição da translucência nuca Ultrassonografia pré-natal

Primeiro trimestre da gravidez Anormalidades congênitas Cardiopatias congênitas

Cariótipo

Desenvolvimento infanti

Keywords

Nuchal translucency measurement Ultrasonography, prenatal Pregnancy trimester, first Congenital abnormalities Heart defects, congenital Karyotype

Child developmen

\title{
Evolução perinatal e pediátrica de crianças com translucência nucal aumentada e cariótipo normal
}

\author{
Perinatal and pediatric follow up of children \\ with increased nuchal translucency and normal karyotype
}

\section{Resumo}

OBJETIVOS: Analisar o desfecho perinatal e pediátrico de fetos que apresentaram translucência nucal (TN) acima do percentil 95 (P95) e cariótipo normal a fim de obter dados que permitam um melhor aconselhamento materno pré-natal. METÓDOS: Análise de fetos em um serviço terciário de obstetrícia apresentaram TN acima do P95 e cariótipo normal entre os anos 2005 e 2011 . Analisamos o seguimento ultrassonográfico gestacional, ecocardiografia (ECO) fetal e pós-natal, peso, comprimento e escore de Apgar ao nascimento, além do desenvolvimento neuropsicomotor por meio do Ages and Stages Questionnaire (ASQ) até jutho de 2012. RESULTADOS: Durante esse período, foram 116 casos de translucência nucal acima do P95, sendo que em 79 (68\%) foi realizado cariótipo fetal. Das análises, 43 foram normais $(54,4 \%)$ e 36 , alteradas $(45,6 \%)$. Nos fetos com cariótipo normal, houve um abortamento na $15^{\text {a }}$ semana gestacional com pentalogia de Cantrel, um óbito na $24^{a}$ semana com diversas anomalias estruturais, um óbito neonatal sem causa definida e dois casos de comunicação intraventricular (CIV) detectados no ECO fetal. Na avaliação ecocardiográfica pós-natal, persistiu um caso de CIV e foi diagnosticado um caso de comunicação interatrial (CIA) e persistência do canal arterial (PCA). Entre os 40 casos sobreviventes, apenas 1 criança apresentou atraso no desenvolvimento da fala e outra apresentou quadro de autismo. Os demais casos resultaram em desenvolvimento neuropsicomotor normal. CONCLUSÃO: No acompanhamento dos fetos com TN aumentada e cariótipo normal, os pais podem ser mais bem aconselhados de que, frente a um exame morfológico-ecocardiográfico do $2^{\circ}$ trimestre sem alterações, a probabilidade de a criança nascer viva e bem é alta $(93,5 \%)$.

\section{Abstract}

PURPOSE: To analyze the perinatal and pediatric outcome of fetuses that showed nuchal translucency (NT) above the 95th percentile (P95) and a normal karyotype in order to obtain data allowing better maternal prenatal counseling. METHODS: fetuses from a tertiary obstetric service with an NT above P95 and a normal karyotype were analyzed between 2005 and 2011 . We analyzed gestational ultrasound follow-up, fetal and postnatal echocardiography (ECHO), weight, length and Apgar score at birth, and neuropsychomotor development by the Ages and Stages Questionnaire (ASQ) up to July 2012. RESULTS: During this period, there were 116 cases of nuchal translucency above the 95th percentile, and the fetal karyotype was determined in 79 of them $(68 \%)$. Forty-three analyses were normal (54.4\%) and 36 were altered (45.6\%). Among the fetuses with a normal karyotype, one was miscarried at 15 weeks of gestation with Cantrel pentalogy and one died at 24 weeks with several structural abnormalities. There was one neonatal death of unknown cause and two cases of intraventricular communication (IVC) detected by fetal ECHO. Postnatal echocardiography revealed the persistence of IVC in one case and one case of atrial septal defect (ASD) and patent ductus arteriosus (PDA). Of the 40 surviving children, only 1 showed delayed speech development and another presented autism. The remaining cases resulted in normal neurodevelopment. CONCLUSION: During the monitoring of fetuses with increased NT and a normal karyotype, parents can be best advised that when a $2^{\text {nd }}$ trimester morphologicalechocardiography ultrasound study is normal, the probability of the child being born alive and well is high (93.5\%).

Correspondência

Sônia Valadares Lemos Silvo

Setor de Obstetrícia

Rua Pedro de Toledo, $1800,5^{\circ}$ andar - Vila Clementino

(EP: 04039-901

São Paulo (SP), Brasil

Recebido

$01 / 03 / 2013$

Serviço de Ginecologia e Obstetrícia do Hospital do Servidor Público Estadual "Francisco Morato de Oliveira" - HSPE-FMO São Paulo (SP), Brasil.

'Serviço de Ginecologia e Obstetrícia, Hospital do Servidor Público Estadual "Francisco Morato de Oliveira" - HSPE-FMO São Paulo (SP), Brasil.

2Setor de Medicina Fetal do Serviço de Ginecologia e Obstetrícia, Hospital do Servidor Público Estadual "Francisco Morato de Oliveira" - HSPE-FMO - São Paulo (SP), Brasil.

3Setor de Obstetrícia do Serviço de Ginecologia e Obstetrícia, Hospital do Servidor Público Estadual "Francisco Morato de Oliveira" HSPE-FMO - São Paulo (SP), Brasil. 
Introdução

A partir do início da década de 1990, vários trabalhos associaram a presença de uma quantidade anormal de fluido na região da nuca fetal, no primeiro trimestre da gestação [translucência nucal (TN) aumentada], com um aumento do risco de cromossomopatias ${ }^{1-8}$. Diferentes mecanismos fisiopatológicos foram propostos para explicar uma TN aumentada no fim do primeiro trimestre da gestação, entre eles as disfunções cardíacas, as anormalidades na matriz extracelular, o desenvolvimento anormal do sistema linfático, as limitações dos movimentos fetais, a anemia fetal ou a hipoproteinemia e as infecções fetais ${ }^{5,9,10}$.

Posteriormente, a medida da TN no primeiro trimestre da gestação passou a ser utilizada como método de rastreamento para cromossomopatias, podendo identificar cerca de $80 \%$ dos fetos acometidos para um falso positivo de $5 \%$ 6-8,11. Quando utilizada juntamente com a idade materna e marcadores bioquímicos do primeiro trimestre, tem uma sensibilidade de $90 \%$, passando a $95 \%$ quando acrescida da avaliação do osso próprio do nariz, para um falso positivo de $5 \%^{12-14}$.

A TN está aumentada em cerca de $4,5 \%$ dos fetos cromossomicamente normais ${ }^{15}$, os quais permanecem, porém, com risco elevado de defeitos estruturais, principalmente cardíacos, e resultados gestacionais adversos, como abortamento, óbito fetal ou neonatal, síndromes genéticas e atraso no desenvolvimento neurológico ${ }^{16-22}$. Em um estudo com 179 fetos com o cariótipo normal e a TN acima do percentil 99, foram encontradas, entre os 162 nascidos vivos acompanhados até os 2 anos de idade, alterações em $11 \%$, sendo metade com defeitos $\operatorname{cardíacos}^{23-25}$. Outro estudo que analisou 451 fetos com o cariótipo normal e a TN acima do percentil 95 (P95) detectou $19 \%$ de resultados adversos ${ }^{18}$. Em 834 fetos com a $\mathrm{TN} \geq 3,0 \mathrm{~mm}$ em parte deles e acima do P95 na parte restante, encontrou-se $11 \%$ de resultados adversos ${ }^{20}$.

Uma recente revisão sistemática observou que $44 \%$ dos fetos com cariótipo normal e defeitos cardíacos congênitos tinham a TN acima do percentil 95 e $20 \%$, acima do percentil $99^{23}$. Em fetos euploides, quanto maior a TN, maior a probabilidade de resultados adversos perinatal, variando de $8 \%$ nos casos de TN no P95-3,4 mm a 80\% nos casos de $\mathrm{TN} \geq 6,5 \mathrm{~mm}^{18}$. O número de anormalidades relacionadas ao aumento da TN não é conhecido. Entretanto, grande parte dos fetos com TN aumentada se mostram neonatos saudáveis $^{26}$. Portanto, TN aumentada não significa necessariamente anormalidade fetal e, sim, risco aumentado para tal.

Alguns autores têm relacionado casos de TN aumentada com déficit no desenvolvimento neurológico pós-natal ${ }^{16,27,28}$, mas em uma revisão recente, os autores concluíram que em fetos com TN aumentada, cariótipo normal e exame ecográfico morfológico normal, o risco de déficit no desenvolvimento neurológico pós-natal não é superior ao da população geral ${ }^{29}$.

O presente estudo tem como objetivo a avaliação pré e pós-natal dos casos com TN aumentada e cariótipo normal em um serviço terciário da região sudeste do Brasil, visando ao aconselhamento dos casais a respeito do resultado do exame.

\section{Métodos}

Foi realizado um estudo retrospectivo no setor de medicina fetal do serviço de Ginecologia e Obstetrícia do Hospital do Servidor Público Estadual "Francisco Morato de Oliveira" (HSPE-FMO) durante o período compreendido entre janeiro de 2005 e dezembro de 2011. O estudo foi aprovado pelo Comitê de Ética da Instituição.

Durante esse período, as gestantes da população geral foram submetidas à avaliação ultrassonográfica de rotina para o rastreamento de aneuploidias entre a $10^{\mathrm{a}}$ e a $14^{\mathrm{a}}$ semanas, e a TN foi medida seguindo as normas recomendadas pela Fetal Medicine Foundation (FMF), de Londres (www.fetalmedicine.com), quando o comprimento cabeça-nádega fetal (CCN) encontrava-se entre $45-84 \mathrm{~mm}$. A TN aumentada foi definida como uma medida acima do percentil 95 para o CCN, segundo a curva de Wright et al ${ }^{30}$. Após o cálculo do risco ajustado (idade materna, $\mathrm{CCN}$ e TN), quando ele se encontrava $\leq 1: 100$, foi oferecida a pesquisa citogenética à paciente. Esta foi realizada nos casos com consentimento pela biópsia de vilo corial (até a $14^{a}$ semana) ou amniocentese (após a 16ª semana). Um exame ultrassonográfico foi realizado pela via transabdominal (transdutor multifrequencial de 3,0-6,0 Mhz, Toshiba Xario, Toshiba medical do Brasil), e por meio de um corte sagital do feto, foi medido o CCN e a espessura máxima da TN.

Nos casos com TN aumentada, as pacientes foram encaminhadas para a realização de ecocardiografia fetal, exame morfológico entre a $20^{\mathrm{a}}$ e a $24^{\mathrm{a}}$ semana, e nova avaliação morfológica no terceiro trimestre (entre a $28^{a}$ e a $34^{\mathrm{a}}$ semana).

Os resultados pós-natal foram obtidos pela análise dos prontuários e/ou por contatos telefônicos. No nascimento, foram analisados a idade gestacional, o peso, o comprimento e o índice de Apgar no primeiro e quinto minutos. O seguimento pós-natal de rotina foi realizado pelo pediatra e teve como objetivo avaliar o crescimento pós-natal, habilidades psicomotoras e da fala, bem como a interação com a criança. Características associadas a síndromes genéticas foram também investigadas. $\mathrm{O}$ acompanhamento variou de seis meses a sete anos, e os seus dados foram obtidos por meio da análise dos prontuários. A avaliação do desenvolvimento neuropsicomotor se deu por intermédio do Ages and Stages Questionnaire (ASQ), que foi respondido pelas mães por contato telefônico. 
O ASQ foi desenvolvido em 1980 como uma ferramenta de triagem para ser respondida pelos pais com o objetivo de detecção precoce dos problemas de desenvolvimento infantil. Ele consiste em uma série de 19 questionários que compreendem o período de desenvolvimento entre 4 meses e 5 anos de idade. Cada questionário contém um conjunto de 30 perguntas que representam 5 domínios: comunicação, atividades motoras grossas e finas, resolução de problemas e habilidades sociais ${ }^{31}$. Em 27 crianças (64\%) foi realizada ecocardiografia pós-natal.

Para a comparação entre proporções, fez-se uso do teste $\chi^{2}$ ou teste exato de Fisher, e, para a comparação entre as médias, foi utilizado o teste $t$ de Student ou o teste não-paramétrico de Mann-Whitney. O nível de significância utilizado para os testes foi de $5 \%$.

\section{Resultados}

Das 3.014 pacientes que foram submetidas à avaliação ultrassonográfica de rotina para o rastreamento de aneuploidias entre a $10^{\mathrm{a}}$ e a $14^{\mathrm{a}}$ semanas, a idade materna variou de 14 a 47 anos, com média de 32 anos, e o CCN variou de 45 a $84 \mathrm{~mm}$, com média de $64 \mathrm{~mm}$, correspondendo a 12 semanas e 5 dias de gestação (variação de 10 a 14 semanas). Dessa amostra, 116 pacientes apresentavam a TN acima do P95 (3,8\%). Em 79 delas, o cariótipo era conhecido. Destas, $43(54,4 \%)$ apresentavam cariótipo normal e $36(45,6 \%)$ alterado.

Entre os cariótipos alterados, observaram-se 14 casos de trissomia do cromossomo 21, 9 de trissomia do 18; 3 de trissomia do 13; 2 casos de monossomia X; 2 casos de $47 \mathrm{XXX}$ e um caso de cada das seguintes: 1 de mosaico do 18, 69XXX, 45XY,der(13;14)(q10;q10), 1 caso de 46XY,13 ps+pstk+, 46XY,del(6)(q25) e 46XY,9ph[14]. Destes, $89 \%$ apresentavam a TN $\geq 3 \mathrm{~mm}$ (Tabela 1 ).

A média de idade das pacientes com TN aumentada foi de 33 anos (variação de 18 a 45 anos e desvio padrão (DP) $=7$ anos), sendo 32 anos no grupo com cariótipo normal (variação de 19 a 43 anos, $\mathrm{DP}=6$ anos) e 35 anos no grupo com cariótipo alterado (variação de 17 a 46 anos, $\mathrm{DP}=8$ anos). Pelo teste $t$ de Student, observou-se que os grupos não diferem em relação à idade $(\mathrm{p}=0,14)$.

A média das medidas das $116 \mathrm{TN}$ aumentadas foi de 3,8 $\mathrm{mm}$ (variação de 2,2 a 17,1 e DP=2,0), sendo 5,5 mm (variação de 2,0 a 16,0 mm e $\mathrm{DP}=3,7$ ) no grupo com cariótipo alterado e 3,5 mm (variação de 2,3 a $8,1 \mathrm{~mm}$ e $\mathrm{DP}=1,4)$ no grupo com cariótipo normal. Pelo teste não-paramétrico de Mann-Whitney, observou-se que os grupos diferem em relação ao valor da TN $(\mathrm{p}<0,05)$. O grupo com cariótipo alterado apresenta maior valor de TN do que o grupo com cariótipo normal.

Dos casos com cariótipo normal, 97,7\% (42/43) foram submetidos à ultrassonografia de segundo trimestre, e somente um caso não participou devido a abortamento espontâneo na $15^{\mathrm{a}}$ semana de gravidez. Dos 42 casos restantes, em 73,8\% (31/42) foram registrados achados normais e em $26,2 \%$ (11/42) foram achados ultrassonográficos anormais (Tabela 2). Entre os 11 casos com alteração morfológica, $45,4 \%$ deles (5/11) foram malformações

Tabela 1. Cariótipos alterados relacionados com o valor da translucência nucal maior que $3 \mathrm{~mm}$

\begin{tabular}{lcc}
\hline Cariótipo & Fetos (n) & TN $\geq 3,0$ mm (n) \\
\hline Trissomia do cromossomo 21 & 14 & 13 \\
Trissomia do cromossomo 18 & 9 & 8 \\
Trissomia do cromossomo 13 & 3 & 3 \\
Síndrome de Turner & 2 & 2 \\
47 XXX & 2 & 2 \\
$45, X Y, 13$ os+pstk+ & 1 & 1 \\
$46, X Y, d e l(6)(q 25)$ & 1 & 0 \\
$46, X Y 9 p h(14)$ & 1 & 0 \\
Tołal & 36 & 32 \\
\hline
\end{tabular}

Tabela 2. Valores descritivos das variáveis de estudo: média (DP) para variáveis quantitativas e frequência absoluta (\%) para variáveis qualitativas

\begin{tabular}{|c|c|c|c|}
\hline Variável & & Classificação & $\begin{array}{c}\text { Valor descritivo } \\
\text { n (\%) }\end{array}$ \\
\hline & Normal & Normal & $31(72,1)$ \\
\hline \multirow[t]{6}{*}{ USG $2^{\circ}$ trimestre } & $M C$ & EPN & $7(16,3)$ \\
\hline & Malformações & MFM & $2(4,8)$ \\
\hline & & $\mathrm{PC}$ & $1(2,4)$ \\
\hline & & RMU & $1(2,4)$ \\
\hline & & CIV & $1(2,4)$ \\
\hline & & Normal & $40(95,2)$ \\
\hline \multirow[t]{2}{*}{ ECO fetal } & & CIV & $1(2,4)$ \\
\hline & & Ventrículo único & $1(2,4)$ \\
\hline Seguimento (meses) & & & $39,7(18,8)$ \\
\hline $\begin{array}{l}\text { IG nascimento-semanas } \\
\text { (média e DP) }\end{array}$ & & & $38,5(2,0)$ \\
\hline Apgar $(1) \geq 7$ & & & $36(87,8)$ \\
\hline Apgar (5) $\geq 7$ & & & $40(97,6)$ \\
\hline Peso g (média e DP) & & & $3166(509,5)$ \\
\hline \multirow[t]{4}{*}{ Comprimento cm (DP) } & & & $47(3,1)$ \\
\hline & & Normal & $38(88,5)$ \\
\hline & & Abortamento & $1(2,3)$ \\
\hline & & Autismo & $1(2,3)$ \\
\hline \multirow[t]{4}{*}{ Desfechos } & $\begin{array}{l}\text { Atraso na } \\
\text { fala }\end{array}$ & $1(2,3)$ & \\
\hline & & $\begin{array}{c}\text { Óbito } \\
\text { intrauterino }\end{array}$ & $1(2,3)$ \\
\hline & & Óbito neonatal & $1(2,3)$ \\
\hline & & Normal & $23(92,0)$ \\
\hline \multirow[t]{2}{*}{ ECO pós-natal } & & CIA e PCA & $1(4,0)$ \\
\hline & & CIV & $1(4,0)$ \\
\hline
\end{tabular}

USG: Ultrassonografia; MC: Marcadores para cromossomopatias: ECO: ecocardiografia; EPN: Espessamento da prega nucal; MFM: Malformações múltiplas: PC: Pentalogia de Cantrel; RMU: Rim multicístico unilateral: CIV: Comunicação interventricular; IG: Idade gestacional; CIA: Comunicação interatrial; PCA: Persistência do canal arterial. 
maiores detectadas no período pré ou pós-natal, dos quais $80 \%$ (4/5) cardíacas.

Em todos os 42 casos, realizou-se ecocardiografia fetal pré ou pós-natal. Destes, 90,4\% foram normais (38/42) e 9,5\% (4/42) apresentaram alterações: 2 casos de comunicação interventricular (CIV), 1 ventrículo único e 1 de associação de persistência do canal atrial (PCA) com comunicação interatrial (CIA).

A evolução completa da gestação foi conhecida em todos os casos com cariótipo normal. Dos $72 \%$ dos casos (31/43) com exame morfológico-ecocardiográfico normal, 93,5\% (29/31) resultaram em nascidos vivos e bem. Os outros 6,5\% (2/31) tiveram desfechos desfavoráveis, dos quais 2 casos apresentavam associação entre alteração no neurodesenvolvimento e defeitos estruturais. Já nos $28 \%$ dos casos com exame morfológico-ecocardiográfico alterado (12/43), 66,7\% (8/12) resultaram em nascidos vivos e bem e $33,3 \%$ (4/12) em desfechos desfavoráveis, sendo 1 caso de abortamento com 15 semanas de gestação (pentalogia de Cantrel), 1 óbito intrauterino com 24 semanas gestacionais (múltiplas malformações na ultrassonografia de primeiro trimestre), 1 óbito neonatal com 27 dias de vida (sem causa conhecida) e 1 caso de CIV (Figura 1).

Todos os 40 sobreviventes tiveram seguimento pós-natal. O tempo médio de acompanhamento foi de 39,7 meses (variação de 6 a 70 meses e $\mathrm{DP}=18,8$ ). A média da idade gestacional no nascimento foi 38,5 semanas (variação de 33 a 42 semanas e $\mathrm{DP}=2,0$ ). A média dos valores de Apgar no $1^{\circ}$ e $5^{\circ}$ minutos foram, respectivamente, 8 (variação de 2 a 9 min e $\mathrm{DP}=1,4$ ) e 9 (variação de 7 a 10 min e $\mathrm{DP}=0,8)$. A média dos pesos ao nascer foi de 3.166 gramas (variação de 1.810 a 4.290 g e $\mathrm{DP}=509,5$ ), e a média das estaturas ao nascer foi de $47 \mathrm{~cm}$ (variação de 41 a $53 \mathrm{~cm}$ e $\mathrm{DP}=3,1$ ).

Alterações do neurodesenvolvimento foram notadas em $5 \%$ dos casos (2/40). Uma criança apresentou atraso no desenvolvimento da fala (que normalizou aos três anos de idade), e uma teve quadro de autismo, ambos diagnosticados aos dois anos de idade. Os demais casos resultaram em desenvolvimento neurológico normal.

A probabilidade de uma gestação com TN aumentada resultar em cariótipo normal é de 68,8\% quando o valor da TN está entre o P95 e 2,9 mm; de 66,6\% quando está entre 3,0 e 3,9 mm; de 37,5\% quando está entre 4,0 e $4,9 \mathrm{~mm}$ e de $28,5 \%$ se o valor for $\geq 5,0 \mathrm{~mm}$. No grupo com cariótipo normal, a probabilidade do exame morfológico-ecocardiográfico ser normal varia de 83,3\% quando a TN está entre 3,0 e 3,9 mm a 50\% quando a TN está $\geq 5,0 \mathrm{~mm}$. Já nos casos nos quais tanto o cariótipo quanto o exame morfológico-ecocardiográfico são normais,

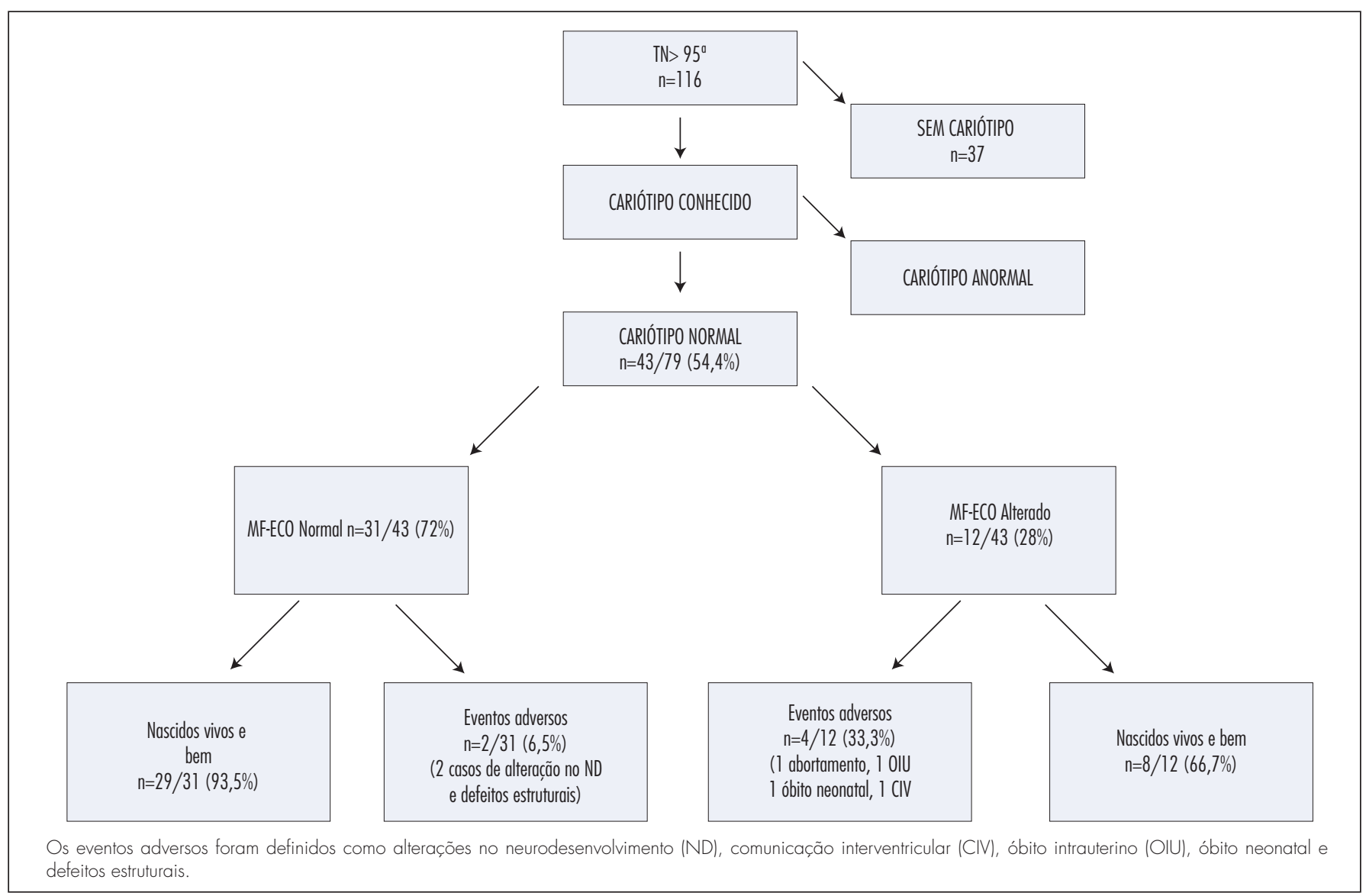

Figura 1. Fluxograma mostrando o desfecho dos fetos com translucência nucal aumentada e cariótipo normal. 
Tabela 3. Probabilidade de cariótipo normal/alterado, exame morfológico-ecocardiográfico normal/alterado e desfecho favorável/adverso em relação ao valor da translucência nucal

\begin{tabular}{|c|c|c|c|c|c|c|}
\hline \multirow{2}{*}{ TN (mm) } & \multicolumn{2}{|c|}{ Cariótipo (\%) } & \multicolumn{2}{|c|}{ Cariótipo normal } & \multirow{2}{*}{$\begin{array}{l}\text { Cariótipo normal / } \\
\text { USG-ECO normal / } \\
\text { desfecho adverso (\%) }\end{array}$} & \multirow{2}{*}{$\begin{array}{l}\text { Cariótipo normal / } \\
\text { USG-ECO normal / } \\
\text { Nascido vivo/bem (\%) }\end{array}$} \\
\hline & Normal & Alterado & Normal & Alterado & & \\
\hline P 95-2,9 & 68,8 & 31,2 & 72,7 & 27,3 & 6,2 & 93,8 \\
\hline $4,0-4,9$ & 37,5 & 62,5 & 66,6 & 33,4 & 0,0 & 100,0 \\
\hline$\geq 5,0$ & 28,5 & 71,5 & 50,0 & 50,0 & 0,0 & 100,0 \\
\hline
\end{tabular}

TN: translucência nucal; P: percentil; USG-ECO: exame morfológico-ecocardiográfico.

a probabilidade dessa gestação resultar em um recém-nascido vivo e bem chega próximo a 100\% (Tabela 3).

Observou-se que quanto maior a TN, maior a razão de probabilidade estimada de cariótipo alterado, sendo $1,1(\mathrm{p}=0,880)$ no intervalo de TN de 3,0 a 3,9 mm; 3,7 $(\mathrm{p}=0,115)$ no intervalo de 4,0 a 4,9 mm e 5,5 (p=0,006) quando a TN é $\geq 5,0 \mathrm{~mm}$, sendo significante nesse caso.

\section{Discussão}

O presente estudo reforça a forte associação existente entre TN aumentada entre 10 a 14 semanas e cromossomopatias. Cerca de $45 \%$ dos casos estudados apresentaram anormalidades cromossômicas, o que está de acordo com Senat et al. ${ }^{32}$, embora haja divergência na literatura ${ }^{6,17-20,33}$. Essa diferença pode ser explicada pelos diferentes pontos de corte utilizados na classificação de TN aumentada e no fato de que neste estudo as pacientes que optaram pela pesquisa do cariótipo fetal apresentavam a TN em um valor mais elevado (média 5,5). Aproximadamente $4 \%$ das pacientes rastreadas para aneuploidias no primeiro trimestre apresentaram TN acima do P95, o que foi concordante com outros estudos ${ }^{17,33}$, porém discordante de Bilardo et al. ${ }^{29}$, que encontraram $8,6 \%$ de TN acima desse percentil. Essa diferença poderia ser explicada pela média de idade mais elevada das pacientes nesse grupo.

Nos casos com cariótipo normal, este estudo confirma a relação entre TN aumentada e anomalias estruturais, principalmente cardíacas e resultados adversos, como abortamento, perda fetal e neonatal. As anomalias estruturais foram detectadas em $9,5 \%$ dos fetos, estando de acordo com outros estudos ${ }^{18-20,33}$, sendo que $80 \%$ delas eram cardíacas. A literatura mostra a predominância de

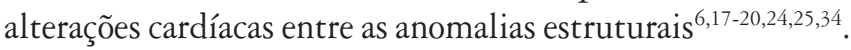
Um importante estudo de revisão mostra que a prevalência de anormalidades fetais maiores na população com TN normal é de $1,6 \%$, havendo uma elevação exponencial com o aumento da TN, sendo 1,5 vezes maior em casos de $\mathrm{TN}$ entre os percentis 95 e $99(2,5 \%)$ e quase 30 vezes maior em $\mathrm{TN} \geq 6,5 \mathrm{~mm}(46,2 \%)^{16}$.
Em relação às perdas fetais, o presente estudo apresentou $4,6 \%$, taxa bem superior à encontrada na população com cariótipo e TN normal $(1,3 \%)^{16}$. Uma revisão recente mostra aumento da taxa de perda fetal e redução da sobrevida com o aumento do valor da TN. Quando a TN encontra-se no intervalo de P95 a 99, a taxa de perda fetal é de $1,3 \%$ e a de RN sem alterações é de $97 \%$. Contudo, quando a TN encontra-se $\geq 6,5 \mathrm{~mm}$, a taxa de perda fetal é elevada a $19 \%$ e a de RN sem alterações, a $15 \%{ }^{16}$.

Apesar de haver um aumento na taxa de resultados adversos nos casos com TN aumentada e cariótipo normal, este estudo demonstrou que a avaliação pós-natal do neurodesenvolvimento, com segmento médio de 3,3 anos, mostrou alguma normalidade em $5 \%$ dos casos, o que não é superior a da população geral. Outros estudos confirmam achado ${ }^{16,18,24,25,29}$. Quanto à avaliação pós-natal, ela foi normal (nascidos vivos e bem) em 92,5\%. Quanto aos resultados adversos, tanto de ordem morfológica quanto funcional, estes ocorreram em 13,9\%, comparado com $11 \%$ no estudo de Ayras et al. ${ }^{20}$, $19 \%$ no de Bilardo et al. ${ }^{18}$, $23 \%$ no de Souka et al. ${ }^{16}$, e $24 \%$ no de Tahmasebpour et al. ${ }^{19}$ No grupo com exame ultrassonográfico pré-natal normal e eventos adversos $(6,5 \%)$, houve 2 casos com alteração no desenvolvimento e defeitos estruturais cardíacos, sendo um caso de autismo e CIV leve diagnosticada em ecocardiografia pós-natal e um caso de atraso na fala e CIA e PCA também diagnosticada no exame pós-natal. Já no grupo com exame ultrassonográfico pré-natal alterado, mas nascidos vivos e bem $(66,7 \%)$, o principal achado foi o espessamento da prega nucal, no segundo trimestre da gestação, sem demais alterações. Uma única criança apresentou rim multicístico unilateral e no pós-natal apresentava vida normal, com o funcionamento adequado do rim contralateral.

Simplificando, este estudo mostrou que um a cada seis fetos vivos, com TN aumentada e cariótipo normal, apresenta algum resultado adverso. Entretanto, quando o seguimento morfológico-ecocardiográfico do segundo trimestre se mostra normal, resultados favoráveis ocorrem em $93,5 \%$ dos casos. 
Os dados deste estudo possibilitaram um melhor aconselhamento aos pais de fetos com TN aumentada e cariótipo normal. Diante deles, pode-se concluir, para a população estudada, que nos casos em que não há alterações adicionais ao acompanhamento ultrassonográfico morfológico-ecocardiográfico, os pais podem ser tranquilizados quanto à alta probabilidade da criança nascer viva e bem $(93,5 \%)$ e à probabilidade de anomalias estruturais residuais e alterações no neurodesenvolvimento não ser maior que na população geral.

\section{Referências}

1. Nicolaides KH, Azar G, Byrne D, Mansur C, Marks K. Fetal nuchal translucency: ultrasound screening for chromosomal defects in first trimester of pregnancy. BM. 1992;304(6831):867-9.

2. Nicolaides $\mathrm{KH}$, Brizot ML, Snijders RJ. Fetal nuchal translucency: ultrasound screening for fetal trisomy in the first trimester of pregnancy. Br J Obstet Gynaecol. 1994; 101(9):782-6.

3. Hinshaw HK. Fetal nuchal translucency: ultrasound screening for fetal trisomy in the first trimester of pregnancy. $\mathrm{Br} \mathrm{J}$ Obstet Gynaecol. 1995; 102(9):758.

4. Cullen MT, Gabrielli S, Green JJ, Rizzo N, Mahoney M, Salafia $\mathrm{C}$, et al. Diagnosis and significance of cystic hygroma in the first trimester. Prenat Diagn. 1990;10(10):643-51.

5. Nicolaides $\mathrm{KH}$, Heath $\mathrm{V}$, Cicero $\mathrm{S}$. Increased fetal nuchal translucency at 11-14 weeks. Prenat Diagn. 2002;22(4):308-15

6. Pandya PP, Snijders R, Johnson SP, De Lourdes Brizot M, Nicolaides $\mathrm{KH}$. Screening for fetal trisomies by maternal age and fetal nuchal translucency thickness at 10 to 14 weeks of gestation. Br J Obstet Gynaecol. 1995; 102(12):957-62.

7. Snijders R, Noble P, Sebire N, Souka A, Nicolaides KH. UK multicentre project on assessment of risk of trisomy 21 by maternal age and fetal nuchal translucency thickness at 10-14 weeks of gestation. Fetal Medicine Foundation First Trimester Screening Group. Lancet. 1998;352(9125):343-6.

8. Taipale P, Hiilesmaa V, Salonen R, Ylostalo P. Increased nuchal translucency as a marker for fetal chromosomal defects. N Engl J Med. 1997;337(23): 1654-8.

9. Haak MC, van Vugt JM. Pathophysiology of increased nuchal translucency: a review of the literature. Hum Reprod Updat. 2003;9(2):175-84.

10. Souka AP, Krampl E, Bakalis S, Heath V, Nicolaides KH. Outcome of pregnancy in chromosomally normal fetuses with increased nuchal translucency in the first trimester. Ultrasound Obstet Gynecol. 2001;18(1):9-17.

1 1. Yoshida S, Miura K, Yamasaki K, Miura S, Shimada T, Tanigawa $T$, et al. Does increased nuchal translucency indicate a fetal abnormality? A retrospective study to clarify the clinical significance of nuchal translucency in Japan. J Hum Genet. 2008;53(8):688-93.

12. Nicolaides KH, Spencer K, Avgidou K, Faiola S, Falcon O. Multicenter study of first-trimester screening for trisomy 21 in 75.821 pregnancies: results and estimation of the potential impact of individual risk-orientated two-stage first-trimester screening. Ultrasound Obstet Gynecol. 2005;25(3):221-6.

13. Nicolaides KH. Nuchal translucency and other first-trimester sonographic markers of chromosomal abnormalities. Am J Obstet Gynecol. 2004;191(1):45-67.

14. Bindra R, Heath V, Liao A, Spencer K, Nicolaides KH. One-stop clinic for assessment of risk for trisomy 21 at 11-14 weeks: a prospective study of 15030 pregnancies. Ultrasound Obstet Gynecol. 2002;20(3):219-25.
15. Nicolaides KH, Brizot ML, Snijders RJ. Fetal nuchal translucency: ultrasound screening for fetal trisomy in the first trimester of pregnancy. Br J Obstet Gynaecol. 1994;101(9):782-6.

16. Souka AP, Von Kaisenberg CS, Hyett JA, Sonek JD, Nicolaides $\mathrm{KH}$. Increase nuchal translucency with normal karyotype. Am J Obstet Gynecol. 2005; 192(4): 1005-21.

17. Saldanha FA, Brizot ML, Moraes EA, Lopes LM, Zugaib M. [Increased fetal nuchal translucency thickness and normal karyotype: prenatal and postnatal follow-up]. Rev Assoc Med Bras. 2009;55(5):57580. Portuguese.

18. Bilardo CM, Muller MA, Pajkrt E, Clur SA, van Zalen MM, Bijlsma EK. Increased nuchal translucency thickness and normal karyotype: time for parental reassurance. Ultrasound Obstet Gynecol. 2007;30(1):11-8.

19. Tahmasebpour A, Rafiee NB, Ghaffari S, Jamal A. Increased nuchal translucency and pregnancy outcome. Iran J Public Health. 2012;41(11):92-7.

20. Ayras O, Tikkanen M, Eronen M, Paavonen J, Stefanovic V. Increased nuchal translucency and pregnancy outcome: a retrospective study of 1063 consecutive singleton pregnancies in a single referral institution. Prenat Diagn. 2013;23:1-7.

21. De Domenico R, Faraci M, Hyseni E, Di Prima FA, Valenti O, Monte $S$, et al. Increased nuchal translucency in normal karyotype fetuses. J Prenat Med. 201 1;5(2):23-6.

22. Alamillo $C M$, Fiddler $M$, Pergament E. Increased nuchal translucency in the presence of normal chromosomes: what's next? Curr Opin Obstet Gynecol. 2012;24(2):102-8.

23. Sotiriadis A, Papatheodorou S, Eleftheriades M, Makrydimas $G$. Nuchal translucency and major congenital heart defects in fetuses with normal karyotype: a meta-analysis. Ultrasound Obstet Gynecol. 2013 Apr 18; [Epub ahead of print]. DOI: 10.1002/ uog. 12488.

24. Senat MV, Frydman R. [Increased nuchal translucency with normal karyotype]. Gynecol Obstet Fertil. 2007;35(6):507-15. French.

25. Senat MV, Bussières L, Couderc $S$, Roume J, Rozenberg $P$, Bouyer $J$, et al. Long-term outcome of children born after a first-trimester measurement of nuchal translucency at the 99th percentile or greater with normal karyotype: A prospective study. Am J Obstet Gynecol. 2007;196(1):53.e1-6.

26. Bekker MN. A normal 20-week scan of a euploid fetus with a history of first-trimester increased nuchal translucency: caution or reassurance? Ultrasound Obstet Gynecol. 2007;30(1):8-10.

27. Mendoza-Caamal EC, Grether-González P, Hernández-Gómez M, Guzmán-Huerta M, Aquinaga-Ríos M. [Birth defects associated with increased nuchal translucency]. Ginecol Obstet Mex. 2010;78(10):533-9. Spanish.

28. Axt-Fliedner R, Hartge D, Chiriac A, Krapp M, Berg C, Geipel $A$, et al. Long-term outcome for children born after a first-trimester measurement of increased nuchal translucency with a normal karyotype: a retrospective analysis. Ultraschall Med. 2009;30(6):558-63. 
29. Bilardo CM, Timmerman E, Paikrt E, van Maarle M. Increased nuchal translucency in euploid fetuses - what should we be telling the parents? Prenat Diagn. 2010;30(2):93-102.

30. Wright D, Kagan KO, Molina FS, Gazzoni A, Nicolaides $\mathrm{KH}$. A mixture model of nuchal translucency thickness in screening for chromosomal defects. Ultrasound Obstet Gynecol. 2008;31(4):376-83.

31. Bricker DD, Squires J, Mounts L. Ages \& Stages Questionnaires (ASQ): a parent-completed child monitoring system. Baltimore: Paul H. Brookes Publishing; 1995.
32. Senat MV, De Keersmaecker B, Audibert F, Montcharmont G, Frydman R, Ville Y. Pregnancy outcome in fetuses with increased nuchal translucency and normal karyotype. Prenat Diagn. 2002;22(5):345-9.

33. Bilardo CM, PajkrtE, de Graaf I, Mol BW, Bleker OP. Outcome of fetuses with enlarged nuchal translucency and normal Karyotype. Ultrasound Obstet Gynecol. 1998;1 1:401-6.

34. Mula R, Goncé A, Bennásar M, Arigita M, Meler E, Nadal A, et al. Increased nuchal translucency and normal karyotype: perinatal and pediatric outcomes at 2 years of age. Ultrasound Obstet Gynecol. 2012;39(1):34-4. 